\title{
Change of International Restless Legs Sy
Study Group Rating Scale subscales with treatment and placebo: a pilot study
}

This article was published in the following Dove Press journal:

Journal of Parkinsonism and Restless Legs Syndrome

2I February 20I4

Number of times this article has been viewed

\author{
Ulrike H Mitchell' \\ Sterling $\mathrm{C}$ Hilton $^{2}$ \\ 'Brigham Young University, \\ Department of Exercise Sciences, \\ ${ }^{2}$ Department of Educational \\ Leadership and Foundations, Provo, \\ UT, USA
}

Background: In 2003, the 10-question International Restless Legs Syndrome Study Group Rating Scale (IRLS) was developed as a means of assessing the severity of restless legs syndrome. Two subscales were identified: symptom severity (SS 1) and symptom impact (SS 2). Only one study has investigated the subscales' responsiveness to a 12-week treatment with ropinirole. This current study was undertaken to assess the impact of a 4-week, non-pharmaceutical treatment on the two subscales and to explore whether or not both subscales were impacted by the observed placebo effect.

Methods: The pooled data from questionnaires of 58 patients (41 from both treatment groups and 17 from the sham treatment control group), who participated in two clinical studies, were reviewed. Their change in score over a 4-week trial was computed. The average change in both subscales in both groups was computed and $t$-tests were performed.

Results: In the treatment group, the average scores of both subscales changed significantly from baseline to week 4 ( $P<0.005$ for both). Compared to the control, SS 1 changed $(P<0.001)$, but not SS $2(P=0.18)$. In the sham treatment group, the scores for SS 1 changed significantly $(P=0.002)$, but not for SS $2(P=0.2)$.

Conclusion: This study corroborated findings from an earlier study in which both subscales changed with a 12-week drug treatment. It also showed that the observed placebo effect is attributed to a small but significant change in symptom severity, but not symptom impact.

Keywords: restless legs syndrome, RLS severity scale, IRLS subscales, symptom impact, symptom severity

\section{Introduction}

There are no definitive biomarkers for restless legs syndrome (RLS). The diagnosis of this neurosensory disorder can therefore only be established based on clinical history and subjective symptoms. Assessing RLS severity and change in symptoms over time is therefore a rather difficult task. In 2003, the International RLS Study Group (IRLSSG) developed the IRLSSG rating scale (IRLS). This 10-item questionnaire was subsequently validated and is now used as a means of assessing the severity of RLS and as a tracking tool for changes in symptoms associated with this pathology. ${ }^{1}$ It consists of questions considering different aspects of the impact of RLS on a patient's life. While validation ${ }^{1}$ and factor analysis ${ }^{2}$ were performed on the original version of the scale, a newer, very similar scale using updated grammar and slightly different wording is the preferred version ${ }^{3}$ (Figure S1). Each question can be answered with a Likert-type scale ranging from "none" (0) to "very severe" (4). Therefore, a maximal score of 40 can be obtained. Generally, an IRLS score between 1 and 10 corresponds
Correspondence: Ulrike H Mitchell Brigham Young University, Department of Exercise Sciences, 106 Smith Fieldhouse, Provo, UT 84602, USA

Tel + I 8014223344

$\mathrm{Fax}+\mathrm{I} 8014220555$

Email rike_mitchell@byu.edu
Journal of Parkinsonism and Restless Legs Syndrome 20I4:4 27-33 
to mild, 11 to 20 moderate, 21 to 30 severe, and 31 to 40 very severe RLS.

Allen et $\mathrm{al}^{2}$ performed a factor analysis of the IRLS in 2003 to evaluate the questionnaire for possible subscales. Over 500 questionnaires were completed over the internet by self-identified RLS patients. Two factors were identified and labeled symptom severity and symptom impact. The symptom severity subscale represents the perceived severity of the disorder and consisted of questions 1, 2, 4, 6,7 , and 8 . The symptom impact subscale corresponds to how the patients see the symptoms affect their lives and consisted of the questions 3, 9, and 10. Question 5, which states "How severe is your tiredness or sleepiness from your RLS symptoms?" loaded equally for both factors and was, therefore, not included in either subscale. The severity factor accounted for $41.8 \%$ and the impact factor for $22.5 \%$ of the overall variance.

Clavadetscher et $\mathrm{al}^{4}$ used the IRLS to track changes in RLS symptoms over a period of 3 years in 70 patients. They found a significant correlation between the improvement of overall clinical impression, as assessed by at least one of the two senior authors, and reduction in the IRLS. The authors therefore suggested that the IRLS might be a reliable instrument for assessing intra-individual changes in RLS severity over time. However, no analysis regarding changes in the two subscales was performed. Many papers have been published since using the change of the IRLS total score as the primary endpoint, but, with the exception of one study, ${ }^{5}$ there are none that analyzed the change in the two subscales.

The study ${ }^{5}$ that analyzed the change in the two subscales used pooled data from the treatment groups of two prior investigations and assessed the reliability, validity, and responsiveness of the IRLS total score and its two subscales on a very large population of RLS patients. The treatment consisted of a once-daily dose with either ropinirole or placebo for 12 weeks. It was confirmed that the questionnaire performed well psychometrically; however, the factor structure found by this group was slightly different from the original study ${ }^{2}$ in terms of its psychometric properties. Item 3 ("Overall, how much relief of RLS arm/leg discomfort do you get from moving around?"), which was originally assigned to the symptom impact subscale was found to measure a "distinct concept, unrelated to the other items in both subscales". Instead, item 5 was found to fit into the symptom impact subscale. The new subscales were found to be valid, reliable and responsive to change; ${ }^{5}$ the symptom severity subscale (SS 1) consists of the following questions (Figure S1): 1, 2, 4, 6, 7, and 8. The symptom impact subscale (SS 2) consists of the following questions: 5,9 , and 10 .

We recently published two studies ${ }^{6,7}$ where we showed significant changes in IRLS score after a 4-week treatment with near-infrared light (NIR), a non-pharmaceutical treatment option. The NIR system is a non-invasive, drug-free device that delivers light at a wavelength of $890 \mathrm{~nm}$ through diodes. ${ }^{8}$ This treatment has been cleared by the US Food and Drug Administration (FDA) for increasing circulation and reducing pain. The proposed mechanism of infra-red light therapy is its ability to generate nitric oxide in the endothelium. ${ }^{9,10}$ Nitric oxide is able to initiate and maintain vasodilation ${ }^{11,12}$ and it influences neurotransmission. ${ }^{12}$ Anecdotal evidence of NIR's ability to decrease symptoms associated with RLS motivated these studies. Based on the literature ${ }^{13-17}$ it can be reasoned that the unpleasant symptoms associated with RLS could be a sign of, or a direct effect from, decreased tissue perfusion. Hence, nitric oxide's chemical property of vasodilation could conceivably explain a temporary decrease in the symptoms associated with RLS.

These previous studies established the following: 1) there is a significant treatment effect of NIR (as assessed by the change in total IRLS within the treatment group and compared to the sham treatment control group); 2) there is a significant placebo effect in the sham treatment control group (as assessed by the change in total IRLS within the control group).

The purpose of this investigation was, therefore, to assess the treatment effect of the 4-week NIR treatment on the two subscales of IRLS and to further explore which of the two subscales responded more strongly to the placebo effect within the control group.

\section{Methods}

IRLS data from two randomized single blind trials were used in this study. The study designs and results for these trials have been described elsewhere. ${ }^{6,7}$ However, to justify combining data from two separate trials, study designs and relevant results will be briefly reviewed. The two studies had several methodological similarities, including inclusion/ exclusion screening criteria, NIR treatment protocol, randomization process, and IRLS data collection. Subjects had to meet the four minimal criteria established by the IRLSSG for the diagnosis of RLS ${ }^{18}$ in order to be admitted to the study and had to score at least 11 points on the IRLS; subjects with decreased sensation were excluded. Subjects received their respective treatment three times a week for 4 weeks; IRLS data were collected before the study began (baseline) and 
at the end of each of the four treatment weeks again. The subjects of both studies were encouraged to maintain their level of medication and to only make changes after confirming with their doctor.

The two studies differed in purpose and, therefore, in treatments administered to subjects. The purpose of the first study $^{6}$ was to assess the effect of NIR treatment on RLS symptoms compared to a sham treatment. Thirty-four subjects participated in the study: 17 were randomly assigned to the NIR treatment and 17 were randomly assigned to the sham treatment. In order to blind the subjects to the treatment, all subjects were set up as if they were receiving the NIR treatment; however, for the sham treatment no actual light energy was administered. The first study found significant improvement in RLS symptoms in both groups; however, the improvement was significantly greater $(P<0.001)$ for the NIR group. Specifically, the NIR group's symptoms improved on average 12 points on the 40 -point IRLS scale while the sham group's improvement was 4.4 points. The statistically significant improvement in the sham group's symptoms is called "the observed placebo effect" because amelioration of symptoms occurred without deliverance of any specific treatment constituents. ${ }^{19}$

The purpose of the second study ${ }^{7}$ was to compare two variations of NIR treatment on RLS symptoms. Twenty-four subjects participated in the study: 13 were randomly assigned to variant 1 of NIR and 11 were randomly assigned to variant 2 of NIR; therefore, all 24 subjects received NIR treatment. No difference was found between the two NIR treatments' impact on RLS symptoms as measured by the IRLS $(P=0.88)$, and both resulted in significant reductions in RLS symptoms $(P<0.001)$ that were similar in magnitude to the treatment effect observed in the first study. ${ }^{6}$

Because the methods and NIR treatment effects were similar between the two trials, IRLS data from both trials are used in this study. Specifically, data from 58 subjects (41 receiving NIR treatment and 17 receiving sham treatment) were analyzed. RLS symptom severity was measured using SS 1 of the IRLS, and RLS symptom impact was measured using SS 2. The changes in RLS symptoms are measured by the changes in IRLS subscale scores between baseline and 4 weeks of treatment. Paired $t$-tests were used to determine significance of changes within a group, and two-sample $t$-tests were used to compare average changes between groups.

\section{Results}

See Table 1 for demographics of the NIR treatment and sham treatment groups.
Table I Demographics of the two groups

\begin{tabular}{lll}
\hline & $\begin{array}{l}\text { NIR treatment } \\
\text { mean (SD) } \\
\mathbf{n = 4}\end{array}$ & $\begin{array}{l}\text { Sham (control) } \\
\text { mean (SD) } \\
\mathbf{n = I 7}\end{array}$ \\
\hline Age (years) & $56.9($ I3.I) & $55.5(I 7 . I)$ \\
Symptom duration (years) & I5.9(I6.0) & $12.7(13.3)$ \\
IRLS baseline score & $23.2(5.5)$ & $23.6(6.9)$ \\
IRLS posttreatment score & II.6 (7.3) & $19.2(7.6)$ \\
IRLS posttreatment change & $-11.6(7.2)$ & $-4.4(3.6)$ \\
Female & $\mathbf{n ~ ( \% )}$ & $\mathbf{n}(\%)$ \\
Positive family history & $24(59)$ & $8(47)$ \\
On medication for RLS & $24(59)$ & $12(7 I)$ \\
\hline
\end{tabular}

Abbreviations: IRLS, International Restless Legs Syndrome Study Group Rating Scale; NIR, near-infrared light; RLS, restless leg syndrome; SD, standard deviation.

In the treatment group, the average scores of both subscales changed significantly from baseline to week 4 $(P<0.005$ for both; Table 2). Compared to controls, there was a significantly greater change in the treatment group in SS $1(P<0.001)$, but no difference in change in SS 2 ( $P=0.178$; Table 3$)$. In the sham treatment group, the scores for SS 1 changed significantly $(P=0.002)$ but not for SS 2 $(P=0.2$; Table 4).

\section{Discussion}

Our study was the first study assessing the within group responsiveness of the two subscales of the IRLS, SS 1 and SS 2, to a 4-week non-pharmaceutical treatment. It confirmed findings from an earlier study ${ }^{5}$ that both subscales are responsive to pharmaceutical treatment. These studies indicate that the subscales respond to pharmaceutical and non-pharmaceutical treatment options.

When comparing responsiveness of the subscales between groups we established that the change in SS 1 was greater in the treatment group compared to the control, but not the

Table 2 IRLS averages per subgroup per question in groups treated with NIR

\begin{tabular}{|c|c|c|c|c|c|}
\hline SS I & Pre & Post & SS 2 & Pre & Post \\
\hline QI & 2.60 & 1.29 & Q5 & 2.00 & 0.90 \\
\hline Q2 & 2.97 & 1.37 & Q9 & 1.55 & 0.60 \\
\hline Q4 & 2.20 & 1.00 & Q10 & 1.53 & 0.60 \\
\hline Q6 & 2.70 & 1.30 & & & \\
\hline Q7 & 3.05 & 1.76 & & & \\
\hline Q8 & 2.20 & 1.16 & & & \\
\hline Average & 2.62 & 1.31 & Average & 1.69 & 0.70 \\
\hline \multicolumn{3}{|c|}{ Paired $t$-test SS I pre/post } & \multicolumn{3}{|c|}{ Paired $t$-test SS 2 pre/post } \\
\hline \multicolumn{3}{|c|}{$P<0.000 I^{*}$} & \multicolumn{3}{|c|}{$P=0.003 *$} \\
\hline
\end{tabular}

Note: *Significant change.

Abbreviations: IRLS, International Restless Legs Syndrome Study Group Rating Scale; NIR, near-infrared light; Q, question; SS I, symptom severity; SS 2, symptom impact. 
Table 3 Average change in subscales

\begin{tabular}{|c|c|c|c|}
\hline & \multirow{2}{*}{$\frac{\text { Treatment with NIR }}{\text { Average (SD) }}$} & \multirow{2}{*}{$\frac{\text { Sham/control }}{\text { Average (SD) }}$} & \multirow{2}{*}{$\begin{array}{l}P \text {-value for two } \\
\text { group comparison }\end{array}$} \\
\hline & & & \\
\hline SS I & 1.31 (0.19) & $0.62(0.26)$ & $0.0004 *$ \\
\hline SS 2 & $0.99(0.09)$ & $0.53(0.48)$ & 0.178 \\
\hline
\end{tabular}

Note: *Significant change.

Abbreviations: NIR, near-infrared light; SD, standard deviation; SS I, symptom severity; SS 2, symptom impact.

change in SS 2. There was no statistically significant change in SS 2 when comparing treatment and control groups. This is probably due to the high variability in the average change of SS 2 in the control group, which is almost the same magnitude as the average itself.

Our study was also the first one exploring whether or not both subscales were impacted by the observed placebo effect. Symptom severity could be defined as the person's disease-specific health status. Its subscale (SS 1) includes symptom related questions on frequency and intensity. Symptom impact could be defined as the person's disease-specific quality of life or functional status. Its subscale (SS 2) includes questions on tiredness/sleepiness from RLS symptoms, their influence on the person's ability to carry out activities of daily living and their negative effect on mood. Having two subscales allows for distinction between the patient's perceived severity of RLS symptoms and the perception of how the symptoms impact life or functioning. ${ }^{2}$ It also allows for an interesting analysis of which of the two subscales is influenced more by the placebo effect. Placebos, "dummy treatments", ${ }^{20}$ or "imitations of specific treatments with the absence of the specific therapeutic constituents" ${ }^{19}$ have been hailed because of their potential therapeutic effect ${ }^{21}$ and discounted on grounds of being false impressions or due to mere psychosomatic effects. ${ }^{19}$ Placebos are most often used when evaluating the efficacy of pharmaceuticals. However, as

Table 4 IRLS averages per subgroup per question in sham treatment control group

\begin{tabular}{llllll}
\hline SS I & Pre & Post & SS 2 & Pre & Post \\
\hline Q1 & 3.00 & 2.20 & Q5 & 2.07 & 1.00 \\
Q2 & 2.90 & 2.40 & Q9 & 1.80 & 1.40 \\
Q4 & 2.00 & 1.70 & Q10 & 1.80 & 1.67 \\
Q6 & 2.80 & 2.10 & & & \\
Q7 & 3.00 & 2.00 & & & \\
Q8 & 2.40 & 2.00 & & \\
Average & 2.57 & 2.03 & Average & 1.89 & 1.36 \\
Paired $t$-test SS I pre/post & & Paired $t$-test pre/post & \\
P=0.002* & & & $P=0.196$ & & \\
\hline
\end{tabular}

Note: *Significant change.

Abbreviations: IRLS, International Restless Legs Syndrome Study Group Rating Scale; Q, question; SS I, symptom severity; SS 2, symptom impact.
Kaptchuck explains in his essay, ${ }^{20}$ they also have their place in alternative medicine. He points out that the placebo effect can be even stronger in alternative medicine because of the different patient and practitioner characteristics. In our studies, all subjects were required to come to a laboratory setting three times a week for 4 weeks. This provided a more intense interaction between them and the investigator as well as the setting in which the treatment took place.

We found that SS 1, measuring symptom severity, significantly changed due to the placebo effect, but not SS 2, measuring the impact RLS symptoms have on RLS sufferers. As noted above, the high variability in the average of this subscale's change probably plays a large role. However, we should ask ourselves, why did we find this high inconsistency, especially when contrasting this to the very low variability exhibited in the treatment group? These findings could result from different causes: 1) some of the social and environmental factors that were used in the study affected the patients' impressions of their disease-specific health status, but not so much the disease-specific quality of life; 2) there could potentially be a delay between the changes in subscales when improvement is due to a placebo effect. This delay is reasonable, taking into account that RLS co-occurs often with anxiety or depressed feelings that can leave the RLS sufferer with a quality of life equivalent to or worse than other major chronic medical disorders. ${ }^{22} \mathrm{~A}$ relatively brief 4 -week trial might not be enough time to help improve these emotions and mend damaged relationships at home and at work; 3 ) the change in severity was present, but too small to obtain an observed reduction in symptom impact. However, the data collected in this study don't allow us to identify the causal mechanism of the observed placebo effect.

The effectiveness of any treatment is generally related to etiology of the condition. RLS etiology is classified into primary (genetic or idiopathic) and secondary, the latter being related to other medical or neurological disorders. ${ }^{23}$ While there is no standard assessment for determining RLS etiology, patients with a positive family history of RLS and no abnormal blood work are generally classified as having primary RLS. Patients without a family history of RLS, with low serum iron levels ${ }^{13,24-27}$ or known pathologies associated with RLS ${ }^{26,28,29}$ are generally classified having secondary RLS. RLS etiology is therefore associated with the effectiveness of pharmacological treatments for RLS; however, etiology was not associated with the effectiveness of NIR (non-pharmacological) in either of the two studies which provided data for this study. 
Our sham-treated control group demonstrated a statistically, but probably not a clinically significant improvement of 4.4 points. The magnitude of our observed placebo effect is relatively small compared to those efficacy trials performed with pharmaceuticals, where the reported improvement, measured by the IRLS, might be around $8^{30}$ to almost $10^{31}$ points. A possible reason for this discrepancy could be the fact that RLS seems to be particularly susceptible to a placebo response because it reacts well to both opioids and dopaminergic (pharmaceutical) agents - two systems associated with the placebo response. ${ }^{32}$ Levine et al ${ }^{33}$ suggested in 1978 that the effect of placebos on pain disorders was mediated, at least partially, by the release of endogenous opioids. Petrovic et a ${ }^{34}$ since confirmed this using tomography by showing increased activity in the anterior cingulate cortex and brainstem during opioid and placebo analgesia. de la Fuente-Fernández et a ${ }^{35}$ and de la Fuente-Fernandez et al and Stoess ${ }^{136}$ found a placebo effect in Parkinson's disease that was related to the release of substantial amounts of endogenous dopamine. However, it is difficult to compare these pharmaceutical placebo responses to the ones found in our study, since most of them are conducted over a 12 -week ${ }^{30,31}$ or even longer trial periods ${ }^{37}$ because the placebo response increases with time..$^{32} \mathrm{~A}$ metaanalysis of the placebo effect in RLS treatment studies ${ }^{32}$ found a substantial placebo response rate, more so for the IRLS than for other IRLS severity measuring tools. It showed a pooled weighted response rate of 40.09 , indicating that about $40 \%$ of the treatment effect was due to a placebo response. The authors point out that the placebo effect for the IRLS was larger than for other RLS scales, and that this was possibly due to its multidimensional features. The meta-analysis ${ }^{32} \mathrm{did}$ not elucidate which of the two subscales was more responsive to the change due to the placebo effect.

In our study we used the subscales as defined by Abetz et $\mathrm{al},{ }^{5}$ which included questions 5,9 , and 10 for SS 2, and not questions 3,9 , and 10 , as originally suggested. ${ }^{2}$ They suggested that, while question 3 ("Overall, how much relief of your RLS arm or leg discomfort do you get from moving around?") was useful as a diagnostic criterion, it might not necessarily be an indicator of symptom severity. Wunderlich et $\mathrm{al}^{38}$ also concluded in their study that question 3 was the most problematic item of the questionnaire. In order to ascertain if the inclusion of questions 3 or 5 in SS 2 would change the results, we repeated our statistical analysis by replacing question 5 by question $3 .^{2}$ Again, the treatment group demonstrated a significant change in SS 2 ( $P=0.002)$, but not the sham treatment group $(P=0.155)$. We also checked if there was a change in SS 2 if we included both questions 3 and 5 in SS 2. Once more, the treatment group demonstrated a significant change $(P=0.002)$, but not the sham treatment group $(P=0.155)$. It can therefore be inferred that, in regards to significance in SS 2, changes in the observed placebo response, it is irrelevant which of the two questions are included in that subscale.

\section{Limitations}

RLS is known to cause significant morbidity, most importantly by way of sleep disruption or sleep deprivation. ${ }^{39}$ Also, or maybe consequently, patients with RLS are likely to have mental health problems, such as anxiety or depressed feelings. ${ }^{40}$ The effectiveness of pharmacological treatments for RLS has been delayed and/or reduced in patients with psychiatric comorbidity ${ }^{41}$ However, no data on the subjects' psychiatric morbidity were collected in either of the two studies from which we pooled the IRLS information for this study. Therefore, we are unable to determine the possible impact of psychiatric comorbidity on the effectiveness of NIR treatment on RLS symptoms as measured by the IRLS subscales.

\section{Author contributions}

UHM: research project conception, organization and execution; statistical analysis execution, review, and critique; manuscript preparation: writing of first draft, review, and critique. SCH: research project organization; statistical analysis review and critique; manuscript preparation: writing of first draft, review, and critique.

\section{Disclosure}

The authors declare no conflicts of interest in this work.

\section{References}

1. The International Restless Legs Syndrome Study Group. Validation of the International Restless Legs Syndrome Study Group rating scale for restless legs syndrome. Sleep Med. 2003;4:121-132.

2. Allen RP, Kushida CA, Atkinson MJ; RLS QoL Consortium. Factor analysis of the International Restless Legs Syndrome Study Group's scale for restless legs severity. Sleep Med. 2003;4(2):133-135.

3. Hening WA, Allen RP. Restless legs syndrome (RLS): the continuing development of diagnostic standards and severity measures. Sleep Med. 2003;4(2):95-97.

4. Clavadetscher SC, Gugger M, Bassetti CL. Restless legs syndrome: clinical experience with long-term treatment. Sleep Med. 2004;5(5): 495-500.

5. Abetz L, Arbuckle R, Allen RP, et al. The reliability, validity and responsiveness of the International Restless Legs Syndrome Study Group rating scale and subscales in a clinical-trial setting. Sleep Med. 2006;7(4): 340-349.

6. Mitchell UH, Myrer JW, Johnson AW, Hilton SC. Restless legs syndrome and near-infrared light: An alternative treatment option. Physiother Theory Pract. 2011;27(5):345-351. 
7. Mitchell UH, Johnson AW, Myrer B. Comparison of two infrared devices in their effectiveness in reducing symptoms associated with RLS. Physiother Theory Pract. 2011;27(5):352-359.

8. Koshi EB, Short CA. Placebo theory and its implications for research and clinical practice: a review of the recent literature. Pain Pract. 2007;7(1):4-20.

9. Matsunaga K, Furchgott RF. Interactions of light and sodium nitrite in producing relaxation of rabbit aorta. J Pharmacol Exp Ther. 1989;248(2):687-695.

10. Mitchell UH, Mack GL. Low-level laser treatment with near-infrared light increases venous nitric oxide levels acutely: a single-blind, randomized clinical trial of efficacy. Am J Phys Med Rehabil. 2013; 92(2):151-156.

11. Ignarro LJ, Buga GM, Byrns RE, Wood KS, Chaudhuri G. Endotheliumderived relaxing factor and nitric oxide possess identical pharmacologic properties as relaxants of bovine arterial and venous smooth muscle. J Pharmacol Exp Ther. 1988;246(1):218-226.

12. Moncada S, Palmer RM, Higgs EA. Nitric oxide: physiology, pathophysiology, and pharmacology. Pharmacol Rev. 1991;43(2):109-142.

13. Ekbom KA. Restless legs syndrome. Neurology. 1960;10:868-873.

14. Lettieri CJ, Eliasson AH. Pneumatic compression devices are an effective therapy for restless legs syndrome: a prospective, randomized, double-blinded, sham-controlled trial. Chest. 2009;135(1):74-80.

15. Rajaram SS, Shanahan J, Ash C, Walters AS, Weisfogel G. Enhanced external counter pulsation (EECP) as a novel treatment for restless legs syndrome (RLS): a preliminary test of the vascular neurologic hypothesis for RLS. Sleep Med. 2005;6(2):101-106.

16. McDonagh B, King T, Guptan RC. Restless legs syndrome in patients with chronic venous disorders: an untold story. Phlebology. 2007;22(4): 156-163.

17. Winkelmann J, Lichtner P, Schormair B, et al. Variants in the neuronal nitric oxide synthase (nNOS, NOS1) gene are associated with restless legs syndrome. Mov Disord. 2008;23(3):350-358.

18. Allen RP, Picchietti D, Hening WA, Trenkwalder C, Walters AS, Montplaisi J; Restless Legs Syndrome Diagnosis and Epidemiology workshop at the National Institutes of Health; International Restless Legs Syndrome Study Group. Restless legs syndrome: diagnostic criteria, special considerations, and epidemiology. A report from the restless legs syndrome diagnosis and epidemiology workshop at the National Institutes of Health. Sleep Med. 2003;4(2):101-119.

19. Kienle GS, Kiene H. The powerful placebo effect: fact or fiction? J Clin Epidemiol. 1997;50(12):1311-1318.

20. Kaptchuk TJ. The placebo effect in alternative medicine: can the performance of a healing ritual have clinical significance? Ann Intern Med. 2002;136(11):817-825.

21. Beecher HK. The powerful placebo. J Am Med Assoc. 1955;159(17): 1602-1606.

22. Abetz L, Allen R, Follet A, et al. Evaluating the quality of life of patients with restless legs syndrome. Clin Ther. 2004;26(6):925-935.

23. Oertel WH, Trenkwalder C, Zucconi M, et al. State of the art in restless legs syndrome therapy: practice recommendations for treating restless legs syndrome. Mov Disord. 2007;22 Suppl 18:S466-S475.
24. Thorpy MJ. New paradigms in the treatment of restless legs syndrome. Neurology. 2005;64(12 Suppl 3):S28-33-S.

25. Sun ER, Chen CA, Ho G, Earley CJ, Allen RP. Iron and the restless legs syndrome. Sleep. 1998;21(4):371-377.

26. Lee KA, Zaffke ME, Baratte-Beebe K. Restless legs syndrome and sleep disturbance during pregnancy: the role of folate and iron. $J$ Womens Health Gend Based Med. 2001;10(4):335-341.

27. Earley CJ, Heckler D, Allen RP. The treatment of restless legs syndrome with intravenous iron dextran. Sleep Med. 2004;5(3):231-235.

28. Appiah-Kubi LS, Pal S, Chaudhuri KR. Restless legs syndrome (RLS), Parkinson's disease, and sustained dopaminergic therapy for RLS. Sleep Med. 2002;3 Suppl:S51-S55.

29. Glasauer FE. Restless Legs Syndrome. Spinal Cord. 2001;39(3): 125-133.

30. Walters AS, Ondo WG, Dreykluft T, Grunstein R, Lee D, Sethi K; TREAT RLS 2 (Therapy with Ropinirole: Efficacy And Tolerability in RLS 2) Study Group. Ropinirole is effective in the treatment of restless legs syndrome. TREAT RLS 2: a 12-week, double-blind, randomized, parallel-group, placebo-controlled study. Mov Disord. 2004;19(12): 1414-1423.

31. Ferini-Strambi L, Aarskog D, Partinen M, et al. Effect of pramipexole on RLS symptoms and sleep: a randomized, double-blind, placebocontrolled trial. Sleep Med. 2008;9(8):874-881.

32. Fulda S, Wetter TC. Where dopamine meets opioids: a meta-analysis of the placebo effect in restless legs syndrome treatment studies. Brain. 2008;131(Pt 4):902-917.

33. Levine JD, Gordon NC, Fields HL. The mechanism of placebo analgesia. Lancet. 1978;2(8091):654-657.

34. Petrovic P, Kalso E, Petersson KM, Ingvar M. Placebo and opioid analgesia - imaging a shared neuronal network. Science. 2002; 295(5560):1737-1740.

35. de la Fuente-Fernández R, Ruth TJ, Sossi V, Schulzer M, Calne DB, Stoessl AJ. Expectation and dopamine release: mechanism of the placebo effect in Parkinson's disease. Science. 2001;293(5532): 1164-1166.

36. de la Fuente-Fernández R, Stoessl AJ. The placebo effect in Parkinson's disease. Trends Neurosci. 2002;25(6):302-306.

37. Trenkwalder C, Benes H, Poewe W, et al; SP790 Study Group. Efficacy of rotigotine for treatment of moderate-to-severe restless legs syndrome: a randomised, double-blind, placebo-controlled trial. Lancet Neurol. 2008;7(7):595-604.

38. Wunderlich GR, Evans KR, Sills T, et al; International Restless Legs Syndrome Study Group. An item response analysis of the international restless legs syndrome study group rating scale for restless legs syndrome. Sleep Med. 2005;6(2):131-139.

39. Hening WA, Allen RP, Chaudhuri KR, et al. Clinical significance of RLS. Mov Disord. 2007;22 Suppl 18:S395-S400.

40. Haba-Rubio J. Psychiatric aspects of organic sleep disorders. Dialogues Clin Neurosci. 2005;7(4):335-346.

41. Hornyak M. Depressive disorders in restless legs syndrome: epidemiology, pathophysiology and management. CNS Drugs. 2010; 24(2):89-98. 


\section{Supplementary materials}

\begin{tabular}{|c|c|}
\hline \multirow[t]{2}{*}{ Item I } & In the past week... \\
\hline & $\begin{array}{l}\text { Overall, how would you rate the RLS discomfort in } \\
\text { your legs or arms? }\end{array}$ \\
\hline \multirow[t]{2}{*}{ Item 2} & In the past week... \\
\hline & $\begin{array}{l}\text { Overall, how would you rate the need to move around } \\
\text { because of your RLS symptoms }\end{array}$ \\
\hline \multirow[t]{3}{*}{ Item 3} & In the past week... \\
\hline & Overall, how much relief of your RLS arm or leg \\
\hline & discomfort did you get from moving around? \\
\hline \multirow[t]{3}{*}{ Item 4} & In the past week... \\
\hline & How severe was your sleep disturbance due to your \\
\hline & RLS symptoms? \\
\hline \multirow[t]{3}{*}{ Item 5} & In the past week... \\
\hline & How severe was your tiredness or sleepiness during the \\
\hline & day due to your RLS symptoms? \\
\hline \multirow[t]{2}{*}{ Item 6} & In the past week... \\
\hline & How severe was your RLS as a whole \\
\hline \multirow[t]{2}{*}{ Item 7} & In the past week... \\
\hline & How often did you get RLS symptoms? \\
\hline \multirow[t]{2}{*}{ Item 8} & In the past week... \\
\hline & $\begin{array}{l}\text { When you had RLS symptoms, how severe were they } \\
\text { on an average? }\end{array}$ \\
\hline \multirow[t]{3}{*}{ Item 9} & In the past week... \\
\hline & Overall, how severe was the impact of your RLS \\
\hline & $\begin{array}{l}\text { symptoms on your ability to carry out your daily affairs, } \\
\text { for example carrying out a satisfactory family, home, } \\
\text { social, school or work? }\end{array}$ \\
\hline \multirow[t]{3}{*}{ Item 10} & In the past week... \\
\hline & How severe was your mood disturbance due to your \\
\hline & $\begin{array}{l}\text { RLS symptoms - for example angry, depressed, sad, } \\
\text { anxious or irritable? }\end{array}$ \\
\hline
\end{tabular}

Figure SI International restless legs syndrome questionnaire.

Note: Adapted from Sleep Medicine, Vol 4, Hening WA, Allen RP, Restless legs syndrome (RLS): the continuing development of diagnostic standards and severity measures, 95-97.' Copyright (C) 2003, with permission from Elsevier.

\section{Reference}

1. Hening WA, Allen RP. Restless legs syndrome (RLS): the continuing development of diagnostic standards and severity measures. Sleep Med. 2003;4(2):95-97.
Journal of Parkinsonism \& Restless Legs Syndrome

\section{Publish your work in this journal}

Journal of Parkinsonism and Restless Legs Syndrome is an online, open access, peer-reviewed journal. The journal publishes review articles, historical reviews, original research articles, case reports, letters to the editor, clinical teaching cases, neuroradiology highlights, neuropathology highlights, neuropsychiatry highlights, autobiographies, conference 